\title{
ANÁLISIS DE LAS POLÍTICAS DE BIOECONOMÍA CIRCULAR EN EUROPA. UN ESTUDIO COMPARATIVO EN REGIONES DEL CENTRO/SUR Y ESTE DE EUROPA
}

\author{
Ignacio Martín-Jiménez ${ }^{a} *$, María Natividad Pérez-Camacho ${ }^{c}$, Ana C. Casillas-González ${ }^{c}$, Manuel \\ García-López $^{b}, \mathbf{M}^{\mathrm{a}}$ Mar Cátedra-Cerón ${ }^{b}$, Carmen Capote ${ }^{b}$, Anastasio J. Villanueva ${ }^{d}$ \\ ${ }^{a}$ CIRCE-Centro de Investigación de Recursos y Consumos Energéticos (Zaragoza, imartin@fcirce.es). \\ ${ }^{b}$ CAGPDS-Consejería de Agricultura, Ganadería, Pesca y Desarrollo Sostenible, Junta de Andalucía \\ (Sevilla, \\ mariam.catedra@juntadeandalucia.es, \\ mcarmen.capote@juntadeandalucia.es,
} manuel.garcia.lo@juntadeandalucia.es)

${ }^{c}$ Tragsatec (Sevilla, acasill1@tragsa.es, mnatipc@gmail.com)

d IFAPA-Instituto de Investigación y Formación Agraria y Pesquera (Granada, anastasioj.villanueva@juntadeandalucia.es)

Resumen: En la última década la bioeconomía circular (BEC) está apareciendo en la agenda política cada vez con más intensidad, tanto a nivel internacional y comunitario, como a nivel nacional y regional. En el presente trabajo se realiza un análisis comparativo de las estrategias relativas a la BEC en una selección de regiones (Flandes, Andalucía, Baden-Württemberg, Pays de la Loire y Mazovia) y países (Italia, Letonia y Reino Unido) de Europa, con el objetivo de identificar aspectos comunes y específicos en cada caso. El criterio utilizado para la selección de casos de estudio fue que la región o país presentase una estrategia de bioeconomía aprobada [a fecha 1/5/2021] y que incluyese un compromiso presupuestario para su implementación. La selección incluye la práctica totalidad de los casos encontrados a nivel europeo que cumplen con este criterio. Del análisis se puede deducir lo que sería una estrategia "tipo", la cual estaría impulsada por la administración pública, implicaría a un amplio espectro de sectores (especialmente sector primario y agroindustrial), y emplearía una variedad de instrumentos de política donde destacarían las acciones de comunicación y concienciación, educación y formación de los trabajadores y empresarios, incentivos positivos (p. ej., ayudas a la $\mathrm{I}+\mathrm{D}+\mathrm{i}$ ), asistencia técnica y de coordinación de iniciativas y simplificación normativa.

Palabras clave: bioeconomía, economía circular, política europea, Pacto Verde, Horizonte Europa.

\section{Introducción}

La bioeconomía circular (BEC) integra los conceptos de bioeconomía y economía circular, con la vocación de representar un modelo económico sostenible económica, social y ambientalmente (Carus y Dammer, 2018; Kardung et al., 2021). La bioeconomía engloba al conjunto de todas las actividades económicas relacionadas con la producción, transformación y utilización, directa o indirecta, de recursos de origen biológico con el fin de producir y transformar biomasa para el suministro de alimentos, piensos, materiales, energía y servicios relacionados con los ciudadanos (EC, 2018). La economía circular es un modelo de producción, distribución y consumo en el que el valor de los productos, materiales y demás recursos permanece el mayor tiempo posible (p. ej., reciclando, reparando, etc.), potenciando su uso sostenible y eficiente y reduciendo al mínimo la generación de residuos (EC, 2015; Ghosh, 2020; Kirchherr et al., 2017). La BEC envuelve, por tanto, elementos comunes a ambos conceptos, como son la mejora del uso de los recursos y la ecoeficiencia, la reducción de la huella de carbono, la reducción de la demanda de carbono fósil, y la valorización de los residuos (Carus y Dammer, 2018).

La BEC implica a múltiples sectores económicos ya existentes, tales como: agricultura, silvicultura, pesca y acuicultura (dentro del sector primario); industria alimentaria, textil, papelera, química, farmacéutica y cosmética, biotecnológica y energética, entre otras (dentro del sector industrial); así como consultoría, logística, etc. (dentro del sector servicios). Por lo tanto, al hablar de sector de la BEC, se consideran aquellas actividades económicas con base biológica que a su vez aplican los principios de la circularidad en sus procesos productivos de forma consecuente. Se puede afirmar que este "nuevo" sector se encuentra en una fase incipiente de desarrollo, en la medida en que está fuertemente sustentado en el conocimiento y la innovación (con lo que ello conlleva en forma de plazos dilatados para el desarrollo y la comercialización de productos). Sin embargo, su expansión es deseable para el conjunto de la sociedad, no solo por la generación de riqueza y empleo asociada a este sector, sino también por los beneficios que genera en forma de externalidades positivas (lucha frente al cambio climático, conservación de recursos naturales, etc.) y minimización de las externalidades negativas (p. ej., asociadas a la generación de residuos). Por ello, el apoyo público para promover el desarrollo del sector de la BEC está justificado tanto desde una perspectiva económica, como medioambiental y social, en la medida en que tal desarrollo derivará en una mejora del bienestar del conjunto de la ciudadanía. 
En la última década, la BEC está adquiriendo mayor presencia en la agenda política, tanto a nivel internacional (OECD, 2018) y comunitario (EC, 2018), como a nivel nacional (MITECO, 2020) y autonómico (p. ej., CAPDR, 2018). Las recientes iniciativas como el Pacto Verde Europeo (EC, 2019) confirman el papel que se espera de la BEC en la Unión Europea (UE) del futuro, así como en cada una de sus regiones. Precisamente, se observa cómo en estas regiones se están comenzando a implementar instrumentos de planificación política (estrategias, planes de acción, etc.) para fomentar el desarrollo de este sector, en buena medida auspiciados por el empuje político mostrado a escala superior. Teniendo en cuenta el reciente advenimiento del concepto de la BEC y de sus conceptos asociados (bioeconomía y economía circular), es útil la comparación entre cómo se han diseñado e implementado estos instrumentos en las regiones de cara a identificar recomendaciones para revisar los existentes y/o mejorar el diseño de los futuros.

En este contexto, el presente estudio tiene por objetivo realizar un análisis comparativo entre instrumentos de planificación política dirigidos al desarrollo de la BEC, especialmente estrategias, en diversas regiones europeas al objeto de apoyar la toma de decisiones públicas orientadas al diseño más eficiente de este tipo de instrumentos. Para ello, se han seleccionado un conjunto de regiones y países europeos, representativos de diversos contextos de implementación.

\section{Metodología}

El estudio está basado en la revisión de las estrategias de BEC o asociadas en una selección de países y/o regiones de Europa. De la revisión se pudo constatar la heterogeneidad de documentos de planificación política (estrategias, hojas de ruta, planes de acción, declaraciones, etc.) y aproximaciones (desde la bioeconomía, la economía circular, así como otras -p. ej., economía verde) existentes a lo largo y ancho del continente. Con objeto de poder realizar un análisis comparativo sistemático de documentos de planificación "comparables" (i.e. con características parecidas), el criterio de selección utilizado fue el de "región o Estado donde existe una estrategia de bioeconomía aprobada [a fecha 1/5/2021], donde exista un compromiso presupuestario para su implementación”. Así, se descartaron regiones y/o Estados con estrategias todavía no aprobadas (caso de algunas regiones del este) o donde no se especificaba el compromiso presupuestario. Así, se identificaron las siguientes ocho regiones y/o Estados que cumplían con estos requisitos: Italia (IT), Letonia (LT), Reino Unido (GB), Flandes (BE), Baden-Württemberg (DE), Andalucía (ES), Pays de la Loire (FR) y Mazovia (PO) $)^{6,7}$.

El análisis se ha circunscrito a los documentos de estrategia si bien la información se ha complementado con otros documentos de planificación (especialmente planes de acción) en los casos en los que estos estaban disponibles (p. ej., Flandes). Para facilitar la consulta de la información extraída, se ha diseñado una tabla con ítems relevantes, que incluye conceptualización e implementación, alcance, instrumentos de política, cuestiones/programas específicos y planificación presupuestaria.

\section{Resultados}

La Tabla 1 muestra de forma esquemática las principales características de las estrategias de bioeconomía analizadas. Si bien todas están enfocadas al desarrollo de la bioeconomía, y la mayoría lo están igualmente respecto de la promoción de modelos de economía circular, la integración de ambos (BEC) solo se menciona expresamente en la mitad de los casos analizados, siendo Andalucía el único caso para el que existe una estrategia expresamente dirigida a la BEC. En todas ellas, participa la administración pública como impulsora, si bien en la mitad (IT, GB, P. Loire y Mazovia) lo hace en colaboración con el sector privado. La mayoría especifica el año 2030 como alcance temporal, seguramente de cara a estar alineados con el horizonte temporal establecido dentro de la UE (EC, 2018). Respecto del alcance sectorial, se observan dos aproximaciones diferenciadas, por una parte estrategias con alcance amplio (Flandes, BadenW., Andalucía y P. Loire) y por otra estrategias con alcance más específico (caso de IT, LV, GB y Mazovia) donde el número de sectores involucrados es sustancialmente menor. En todo caso, los sectores primarios (especialmente agricultura) y bioindustria (en particular, la industria alimentaria) están recogidos en todas las estrategias analizadas, mientras que otros como el de energía están presentes en una amplia mayoría.

Como puede observarse igualmente en la tabla, la variedad de instrumentos de política dirigidos a fomentar el desarrollo de la bioeconomía en cada caso difiere. Exceptuando las acciones relativas a comunicación y concienciación de las iniciativas de bioeconomía y sus beneficios para la sociedad, presentes en todas las estrategias analizadas, el uso del resto de instrumentos no está generalizado. Por ejemplo, en la mitad de las estrategias se emplea contratación pública innovadora (CPI) y fiscalidad verde, capacitación del personal público, instrumentos colaborativos (como clústers o hubs); en cinco de las ocho estrategias, se

\footnotetext{
${ }^{6}$ Finlandia también cumplía con los requisitos, pero se decidió no incluir en el análisis dado que durante el 2021 se espera la nueva estrategia de bioeconomía (que sustituye a la anterior aprobada en 2014).

${ }^{7}$ Los documentos utilizados para el análisis están disponibles online. De cualquier forma, se les puede facilitar para los lectores interesados, bajo petición.
} 
incluyen incentivos positivos, asistencia técnica y acciones de coordinación, simplificación normativa; mientras que en seis se incluyen acciones enmarcadas dentro de la educación y formación de los empresarios y trabajadores. Llama la atención cómo en ningún caso se establece el uso de incentivos negativos (p. ej., impuestos al uso de insumos) como instrumentos relevantes para el desarrollo de la bioeconomía.

Tabla 1. Estrategias de bioeconomía de los casos seleccionados. Elementos comunes relativos a la bioeconomía circular

\begin{tabular}{|c|c|c|c|c|c|c|c|c|c|}
\hline Aspectos & Ítems & IT & $\mathbf{L V}$ & GB & $\begin{array}{l}\text { Flandes } \\
\text { (BE) }\end{array}$ & $\begin{array}{l}\text { Baden- } \\
\text { W. (DE) }\end{array}$ & $\begin{array}{l}\text { Andalu- } \\
\text { cía (ES) }\end{array}$ & $\begin{array}{l}\text { P. Loire } \\
\text { (FR) }\end{array}$ & $\begin{array}{c}\text { Mazovia } \\
\text { (PO) }\end{array}$ \\
\hline \multirow{2}{*}{$\begin{array}{l}\text { Conceptuali- } \\
\text { zación e } \\
\text { implementa- } \\
\text { ción }\end{array}$} & $\begin{array}{l}\text { Bioeconomía } \\
\text { circular }\end{array}$ & $\mathrm{X}$ & & & & $\mathrm{X}$ & $\mathrm{X}$ & $\mathrm{X}$ & \\
\hline & $\begin{array}{l}\text { Tipo de } \\
\text { iniciativa } \\
\text { [impulsor] }\end{array}$ & $\begin{array}{c}\text { PU-PR } \\
\text { (varios } \\
\text { M\%/hubs) }^{\circ} \text { /hubs }\end{array}$ & $\begin{array}{c}\mathrm{PU} \\
\left(\mathrm{M}^{\mathrm{o}}\right. \\
\text { Agric. })\end{array}$ & $\begin{array}{c}\text { PU-PR } \\
\text { (varios } \\
\text { M\%/hubs) }^{\circ}\end{array}$ & $\begin{array}{c}\text { PU } \\
\text { (Gob. } \\
\text { Flandes) }\end{array}$ & $\begin{array}{c}\text { PU } \\
\text { (Gob. B- } \\
\text { W) }\end{array}$ & $\begin{array}{c}\text { PU } \\
\text { (Consej. } \\
\text { Agric.,G. } \\
\text { P.D.S.) }\end{array}$ & $\begin{array}{l}\text { PU-PR } \\
(\mathrm{AC} 3 \mathrm{~A})\end{array}$ & $\begin{array}{l}\text { PR-PU } \\
\text { (agencia } \\
\text { regional } \\
\text { energía) }\end{array}$ \\
\hline \multirow[t]{2}{*}{ Alcance } & Sectores & $\begin{array}{l}\text { A01,A03 } \\
\text {,C }\end{array}$ & $\begin{array}{c}\text { A01- } \\
03, \mathrm{C} 10- \\
\text { 11,C17, } \\
\text { C20,D35 }\end{array}$ & $\begin{array}{c}\text { A01,C1- } \\
\text { 11,C21, } \\
\text { D35,F41 } \\
-42, H,\end{array}$ & $\begin{array}{c}\mathrm{A} 01- \\
03, \mathrm{C} 10- \\
11, \mathrm{C} 13- \\
15, \mathrm{C} 16- \\
17, \mathrm{C} 20- \\
21, \mathrm{C} 31, \\
\mathrm{D} 35, \mathrm{E} 38 \\
\text {,F41- } \\
42, \mathrm{H} \\
\end{array}$ & $\begin{array}{l}\text { Todos } \\
\text { (ver } \\
\text { nota) }\end{array}$ & $\begin{array}{l}\text { Todos } \\
\text { (ver } \\
\text { nota) }\end{array}$ & $\begin{array}{l}\text { Todos } \\
\text { (ver } \\
\text { nota) }\end{array}$ & $\begin{array}{c}\text { A01- } \\
\text { 03,C20- } \\
\text { 21,D35, } \\
\text { E38 }\end{array}$ \\
\hline & $\begin{array}{l}\text { Marco } \\
\text { temporal }\end{array}$ & & 2030 & 2030 & 2030 & & 2030 & 2021 & 2030 \\
\hline \multirow{8}{*}{$\begin{array}{l}\text { Instrumentos } \\
\text { de política }\end{array}$} & $\begin{array}{l}\text { Incentivos } \\
\text { positivos }\end{array}$ & $X$ & $X$ & & $X$ & $X$ & $X$ & & \\
\hline & $\begin{array}{l}\text { CPI y fiscali- } \\
\text { dad verde }\end{array}$ & & $X$ & & $\mathrm{X}$ & $\mathrm{X}$ & $\mathrm{X}$ & & \\
\hline & $\begin{array}{l}\text { Asist. técnica y } \\
\text { coordinación }\end{array}$ & $X$ & & $\mathrm{X}$ & & $X$ & $\mathrm{X}$ & $X$ & \\
\hline & $\begin{array}{l}\text { Simplificación } \\
\text { normativa }\end{array}$ & & $X$ & $\mathrm{X}$ & $\mathrm{X}$ & $\mathrm{X}$ & & $\mathrm{X}$ & \\
\hline & $\begin{array}{l}\text { Capacitación } \\
\text { personal públi. }\end{array}$ & & & $\mathrm{X}$ & & $X$ & & $\mathrm{X}$ & $\mathrm{X}$ \\
\hline & $\begin{array}{l}\text { Colaborativos } \\
\text { (clúster/hub) }\end{array}$ & $\mathrm{X}$ & & & $\mathrm{X}$ & $\mathrm{X}$ & $\mathrm{X}$ & & \\
\hline & $\begin{array}{l}\text { Comunicación } \\
\text { y concienc. }\end{array}$ & $\mathrm{X}$ & $X$ & $\mathrm{X}$ & $\mathrm{X}$ & $\mathrm{X}$ & $\mathrm{X}$ & $\mathrm{X}$ & $\mathrm{X}$ \\
\hline & $\begin{array}{l}\text { Educación y } \\
\text { formación }\end{array}$ & $X$ & $X$ & & $\mathrm{X}$ & $\mathrm{X}$ & $\mathrm{X}$ & & $\mathrm{X}$ \\
\hline \multirow{4}{*}{$\begin{array}{l}\text { Cuestiones/ } \\
\text { programas } \\
\text { específicos }\end{array}$} & Zonas rurales & $X$ & $X$ & $\mathrm{X}$ & & $\mathrm{X}$ & & & $X$ \\
\hline & Biorrefinerías & $X$ & & $X$ & & $X$ & $X$ & & $X$ \\
\hline & Logística & & & & & & $\mathrm{X}$ & & \\
\hline & $\begin{array}{l}\text { Desperdicio } \\
\text { alimentario }\end{array}$ & $X$ & & $X$ & & & & & $X$ \\
\hline \multicolumn{2}{|c|}{ Planificación presupuestaria } & $\mathrm{X}$ & & $\mathrm{X}$ & $\mathrm{X}$ & & $X$ & $\mathrm{X}$ & $\mathrm{X}$ \\
\hline
\end{tabular}

Nota: Tipo de iniciativa: PU, pública; PR, privada. Sectores, utilizando la clasificación NACE-rev2 (Reglamento (CE) No 1893/2006): Agricultura [A01], Forestal [A02], Pesca y acuicultura [A03], Ind. alimentaria y bebidas [C10,11], Ind. textil (incl. ropa, cuero y calzado) [C13,14,15], Ind. mueble y madera [C16,31], Ind. papelera [C17], Ind. química [C20], Ind. farmacéutica [C21], Productos de plástico y caucho [C22], Energía eléctrica, gas, vapor y aire acondicionado [D35], Suministro y tratamiento de agua [E36,37], Gestión de residuos [E38], Construcción [F41,42], Comercio [G46,47], Transporte y almacenamiento [H], Hostelería [I55,56] 
Por otra parte, cabe comentar ciertas cuestiones específicas relevantes para el adecuado desarrollo de la BEC. Se observa por ejemplo cómo la mayoría de las estrategias incluyen acciones y/o medidas específicamente dirigidas a las zonas rurales. Igualmente, llama la atencion que el fomento de las biorrefinerías no sea una cuestión prioritaria común a todas las estrategias analizadas, aunque se mencione en una mayoría de ellas. En el otro extremo aparecen otros elementos importantes de la BEC pero que reciben una menor atención en las estrategias analizadas, como son la logística (p. ej., solo Andalucía incluye acciones directamente orientadas a mejorar la logística de los subproductos) o el desperdicio alimentario (al que se refieren las estrategias de IT, GB, y Mazovia).

Finalmente, respecto de la financiación de las acciones establecidas en las estrategias, cabe apuntar que en general no se provee de información detallada sobre la planificación presupuestaria (p. ej., montantes presupuestarios y fondos asociados por concepto/partida), si bien en la mayoría de los casos sí se incorporan directrices generales sobre las fuentes de financiación (p. ej., en Flandes en el recientemente aprobado plan de acción se especifica el uso de nuevos fondos, provenientes del Mecanismo Europeo de Recuperación y Resiliencia).

\section{Conclusiones}

La bioeconomía debe adoptar el paradigma de la circularidad en los procesos productivos, maximizando de esa forma su retorno socioeconómico y ambiental. Así, toda estrategia de bioeconomía debe asegurar que promueve de forma efectiva el desarrollo de una BEC. Quizá por lo reciente de ambos conceptos, bioeconomía y economía circular, los cuales todavía no presentan una definición ampliamente aceptada (tanto en el plano académico como técnico), aún no se ha articulado la integración de ambos dentro de la planificación política. Del análisis realizado, que implica una selección de las escasas estrategias de bioeconomía implementadas en las regiones y Estados miembro de la UE, no se puede anticipar que en efecto las futuras estrategias de bioeconomía a niveles regionales y/o nacionales lo serán de BEC. Sin embargo, estrategias nacionales como la de Italia, o regionales como la de Baden-W., Andalucía o P. Loire, todas ellas enfocadas en la BEC, bien de forma tácita (caso de Baden-W.) o expresa (caso de Andalucía), están marcando el camino en esa dirección.

En todo caso, el presente análisis muestra una realidad heterogénea no sólo en lo que se refiere a la propia conceptualización de la BEC, ya comentada, sino también a los diferentes agentes impulsores, alcances sectoriales, usos de instrumentos de política, identificación de elementos clave, entre otros. No obstante, a partir de los resultados del análisis se puede afirmar que una estrategia "tipo" estaría impulsada por la administración pública (p. ej., organismo competente en el sector primario), implicaría a un amplio espectro de sectores (especialmente sector primario y bioindustria), emplearía una variedad de instrumentos de política donde destacarían las acciones de comunicación y concienciación, educación y formación de los trabajadores y empresarios, incentivos positivos (p. ej., ayudas a la I+D+i), asistencia técnica y de coordinación de iniciativas y simplificación normativa.

\section{Agradecimientos}

El trabajo se ha realizado en el marco del proyecto europeo POWER4BIO (https://power4bio.eu; GA 818351). Bibliografía

CAPDR (Consejería de Agricultura, P.y.D.R. (2018). Estrategia Andaluza de Bioeconomía Circular. Consejería de Agricultura, Pesca y Desarrollo Rural, Junta de Andalucía, Sevilla.

Carus, M. y Dammer, L. (2018). The “circular bioeconomy”-Concepts, opportunities and limitations. nova-Institut, Hürth (DE).

EC (European Commission). (2015). Closing the loop - An EU action plan for the circular economy. COM(2015) 614 final. European Commission, Brussels.

EC (European Commission). (2018). A sustainable bioeconomy for Europe: Strengthening the connection between economy, society and the environment. COM(2018) 673 final. European Commission, Brussels.

EC (European Commission). (2019). The European Green Deal. COM(2019) 640 final. European Commission, Brussels.

Ghosh, S.K. (Ed.) (2020). Circular economy: Global perspective. Singapore: Springer Nature.

Kardung, M., Cingiz, K., Costenoble, O., Delahaye, R., Heijman, W., Lovrić, M., . . Zhu, B.X. (2021). "Development of the circular bioeconomy: drivers and indicators". Sustainability, 13(1): 413.

Kirchherr, J., Reike, D. y Hekkert, M. (2017). "Conceptualizing the circular economy: An analysis of 114 definitions”. Resources, Conservation and Recycling, 127: 221-232.

MITECO (Ministerio para la Transición Ecológica y Reto Demográfico). (2020). España Circular 2030. Estrategia Española de Economía Circular. MITECO, Madrid.

OECD (Organization for Economic Cooperation and Development). (2018). Meeting policy challenges for a sustainable bioeconomy. OECD Publishing, Paris. 